\title{
Investigation of the Effect of Grid Size on External Store Separation Trajectory Using CFD
}

\author{
Alaa A. Osman, Amgad M. Bayoumy, Ismail El baialy, Osama E. Abdellatif, Essam E. Khallil
}

\begin{abstract}
In this paper, a numerical simulation of a finned store separating from a wing-pylon configuration has been studied and validated. A dynamic unstructured tetrahedral mesh approach is accomplished by using three grid sizes to numerically solving the discretized three dimensional, inviscid and compressible Euler equations. The method used for computations of separation of an external store assuming quasi-steady flow condition. Computations of quasi-steady flow have been directly coupled to a six degree-offreedom (6DOF) rigid-body motion code to generate store trajectories. The pressure coefficients at four different angular cuts and time histories of various trajectory parameters and wing pressure distribution during the store separation are compared for every grid size with published experimental data.
\end{abstract}

Keywords - CFD Modelling, Quasi-steady Flow, Moving-body Trajectories, Transonic Store Separation, Moving-body Trajectories.

\section{INTRODUCTION}

$\mathrm{S}_{\mathrm{n}}^{\mathrm{A}}$ AFE separation of a store from an aircraft is one of the major aerodynamic problems in the design and integration of a new store to an aircraft. Carriage loads and moments acting on the store should be correctly predicted in order to have an idea about its behavior after separation.

In addition to the wind tunnel [1], [2] and flight test [3], the computational simulation has been recently used to reduce cost and time incurred to generate and test a particular model. The first step in any flow simulation is the discretization of the physical domain. Many techniques for handling the computational domain with moving boundaries have been devised and are currently being used such as Cartesian approach [4]-[7], overset grids [8]-[16] and dynamic meshes [17]-[27].

The Cartesian approach implies the use of non-body-fitted volume meshes. This method has one major drawback when conventional finite volume procedures are used, accuracy is compromised in the cut cells on the boundaries where any error in these regions leads to inaccurate prediction of performance [28]. In the overset grid, separate grids around the moving bodies are applied and interpolation between these grids and a global grid are updated each step. However, a large

Alaa A. Osman is with the Egyptian Armed Forces, Egypt (e-mail: Alaa.amo@hotmail.com).

Amgad M. Bayoumy is with Military Technical College, Egypt (e-mail: Dramgad@mep-ls.com).

Ismail El Baialy is with the Mechanical Engineering Department, Qatar University, Qatar (Esmai_Bialy@hotmail.com).

Osama E. Abdellatif is with Mechanical Engineering Department, Banha University, Egypt (e-mail: Sameh_Sohasmr@yahoo.com).

Essam E. Khallil is with Mechanical Engineering Department, Cairo University, Egypt (e-mail: khalile1@asme.org). number of cells are used to enhance the resolution of the solution which requires extra memory and computational overhead [16]. Dynamic meshes apply deformation of the volume mesh in response to the surface motion. The most significant advantage of utilizing unstructured dynamic meshes is the flexibility to handle complex geometries. Grid generation time is greatly reduced because the user's input is limited to mainly generation of a surface mesh. Finally, because there is no overlapping grid regions, fewer grid points are required [26].

In the present work, quasi-steady approach is used to demonstrate the accuracy and technique of using an unstructured dynamic mesh approach in a store separation study to assess the effect of grid size on store trajectories. The implementation of a 6DOF (six degree of freedom) flight mechanics model for rigid bodies and its coupling to CFD is presented. An inviscid flow is assumed to simplify the above simulations. The predicted computed trajectories are compared with a 1/20 scale wind-tunnel experimental data conducted at the Arnold Engineering Development Center (AEDC) [1] under transonic conditions (Mach number 95) at an altitude of $11,600 \mathrm{~m}$ and $0^{\circ}$ angle of attack for a particular weapon configuration with appropriate ejection forces.

\section{MODEL DESCRIPTION}

\section{A. Wind Tunnel Test Model}

A generic finned-store shape and a clipped delta wing with a $45^{\circ}$ leading edge sweep, a pylon located at the mid span of the wing, and a store having four tail fins positioned in a cruciform style. Wing profile is NACA64A010 airfoil section and is constant throughout the span. Trailing edge has no sweep angle and the taper ratio is 0.133 . The store diameter is 0.5081 meters. The fins are identical and they have a constant profile NACA0008 throughout the fin span. Leading and trailing edge sweep angles of the fins are 60 and 0 degrees, respectively. The sketch of the test case model and the sting used in the wind tunnel test conducted at the Arnold Engineering Development Center (AEDC) [1] are represented in Fig. 1

\section{B. Computational Model}

The computational model geometry is similar to the experimental test specimen. For the sake of the physical coherence, the store is modeled with the sting mounted to the end of the store as it was used in the captive trajectory system experiments. Detailed drawings of the wing and the coordinate system are given in Fig. 2. 


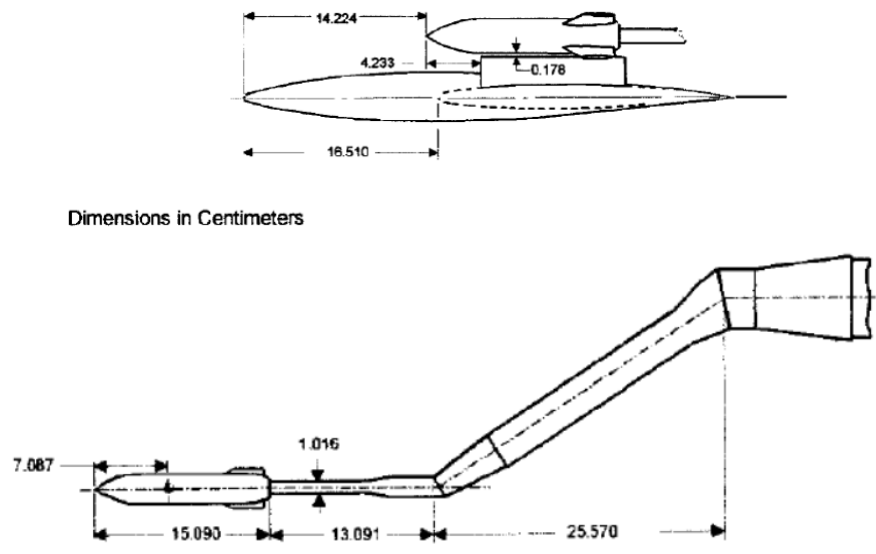

Fig. 1 Model and the sting used in the wind tunnel test (AEDC) [1]

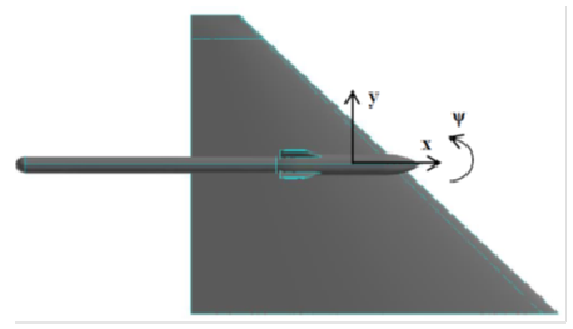

(a) Bottom view

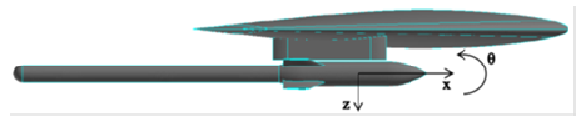

(b) Side view

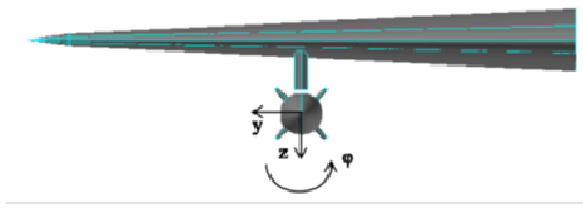

(c) Front view

Fig. 2 Computational model and sign convention

\section{GOVERNING EQUATION AND BOUNDARY CONDITIONS}

\section{A. Euler Equations}

Governing equation of unsteady inviscid flow-field is Euler equation. Euler's equation for inviscid fluid flow is a special/limiting case of the more general non-linear NavierStokes equation - which expresses Newton's 2nd law of motion for compressible fluid flow. The nondimensional conservation law form is

$$
\partial_{t} Q+\partial_{x} E+\partial_{y} F+\partial_{z} G=0
$$

where, $Q$ is the conserved variable,

$$
Q=\left[\begin{array}{c}
\rho \\
\rho u \\
\rho v \\
\rho w \\
e_{t}
\end{array}\right]
$$

$E, F$ and $G$ are the flux vectors,

$$
E=\left[\begin{array}{c}
\rho u \\
\rho u^{2}+p \\
\rho u v \\
\rho u w \\
u\left(e_{t}+p\right)
\end{array}\right], F=\left[\begin{array}{c}
\rho v \\
\rho v u \\
\rho v^{2}+p \\
\rho v w \\
v\left(e_{t}+p\right)
\end{array}\right] \text { and } G=\left[\begin{array}{c}
\rho w \\
\rho w u \\
\rho w v \\
\rho w^{2}+p \\
w\left(e_{t}+p\right)
\end{array}\right]
$$

The total energy per unit mass in the above equations is composed of static and dynamic parts

$$
e_{t}=e+\frac{1}{2}\left(u^{2}+v^{2}+w^{2}\right)
$$

Static energy and pressure can be expressed as a function of local speed of sound, temperature of the fluid and the ratio of specific heats.

$$
e=\frac{a^{2}}{\gamma(\gamma-1)} \quad, \quad p=\frac{\rho a^{2}}{\gamma}
$$

where,

$$
a^{2}=\gamma R T, \quad \gamma=C_{p} / C_{v}
$$

\section{B. Newton-Euler Equations of Motion}

The 6 DOF rigid-body motion of the store is calculated by numerically integrating the Newton-Euler equations of motion within Fluent as a user-defined function (UDF). The aerodynamic forces and moments on the body store are calculated based on the integration of pressure over the surface. This information is provided to the 6DOF from the flow solver in inertial coordinates. The governing equation for the translational motion of the center of gravity is solved for in the inertial coordinate system, as:

$$
\dot{\vec{v}}_{G}=\frac{1}{m} \sum \vec{f}_{G}
$$

$\dot{\vec{v}}_{G}$ is the translational motion of the center of gravity, $m$ is the mass, and $\vec{f}_{G}$ is the force vector due to gravity.

The angular motion of the object, $\dot{\vec{\omega}}_{\mathrm{B}}$ is more easily computed using body coordinates

$$
\dot{\vec{\omega}}_{\mathrm{B}}=\mathrm{L}^{-1}\left(\sum \overrightarrow{\mathrm{M}}_{\mathrm{B}}-\vec{\omega}_{\mathrm{B}} \times \mathrm{L} \vec{\omega}_{\mathrm{B}}\right)
$$

$\mathrm{L}$ is the inertia tensor, $\vec{\omega}_{\mathrm{B}}$ is the rigid body angular velocity vector, and $\overrightarrow{\mathrm{M}}_{\mathrm{B}}$ is the moment vector of the body transformed from inertial to body coordinates using $\overrightarrow{\mathrm{M}}_{\mathrm{B}}=\mathrm{R} \overrightarrow{\mathrm{M}}_{\mathrm{G}}$, where $\mathrm{R}$ represents the following transformation matrix

$$
R=\left[\begin{array}{ccc}
C_{\theta} C_{\psi} & C_{\theta} C_{\psi} & -S_{\theta} \\
S_{\phi} S_{\theta} C_{\psi}-C_{\phi} S_{\Psi} & S_{\phi} S_{\theta} C_{\psi}+C_{\phi} S_{\Psi} & S_{\phi} C_{\theta} \\
C_{\phi} S_{\theta} C_{\psi}+S_{\phi} S_{\psi} & C_{\phi} S_{\theta} C_{\psi}-S_{\phi} S_{\psi} & C_{\phi} C_{\theta}
\end{array}\right]
$$

In generic terms, $C_{x}=\cos (x)$ and $S_{x}=\sin (x)$. The attitude of the separation from aircraft is described by Euler angles, yaw angle psi $(\psi)$, pitch angle theta $(\theta)$ and roll angle phi $(\varphi)$. 


\section{Boundary Conditions}

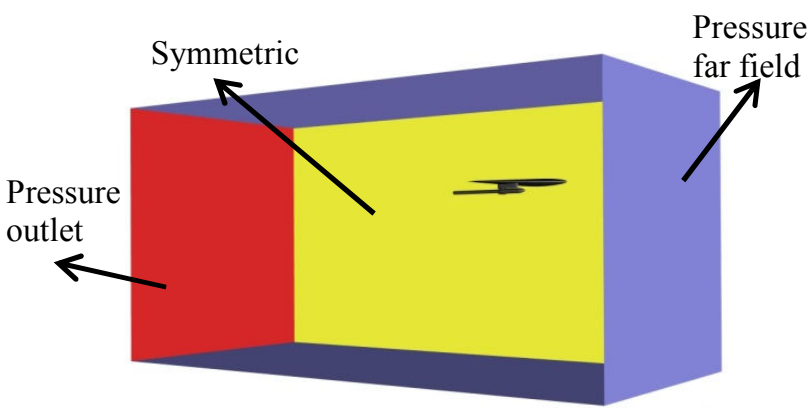

Fig. 3 Solution domain and boundary conditions

The boundary conditions are represented in Fig. 3. All-side boundaries except downstream and the right-side were set as far field, with a standard atmosphere model for $8000 \mathrm{~m}$ altitude temperature and pressure free stream conditions. Downstream and right-side boundaries were defined as pressure outlet and symmetric boundary respectively. The wing, pylon, and store were modeled as solid wall boundary with non-slip velocity.

\section{Computational Grid Generation}

Computational grid generation of the geometry is carried out in Gambit. A dynamic unstructured tetrahedral mesh approach is accomplished by using three grid sizes [coarse grid (1047529), medium grid (2695159) and fine grid (3529136 elements)] to numerically solving and comparing the results of the discretized three dimensional, inviscid and compressible Euler equations with the wind tunnel test of reference [1]. The mesh quality was iteratively improved by varying the grading type, ratio and interval count for the edge meshing with the aim of having a low skewness. Also sizing function was used on faces with small dimensions. It was ensured that no degenerative elements were formed in the mesh and the skewness range was also within limits. Fig. 2 shows a surface meshing of the wing-store configuration. In the figure it can be seen that the mesh is fine close to the wing-store configuration and gets coarser as the distance from the configuration increases.

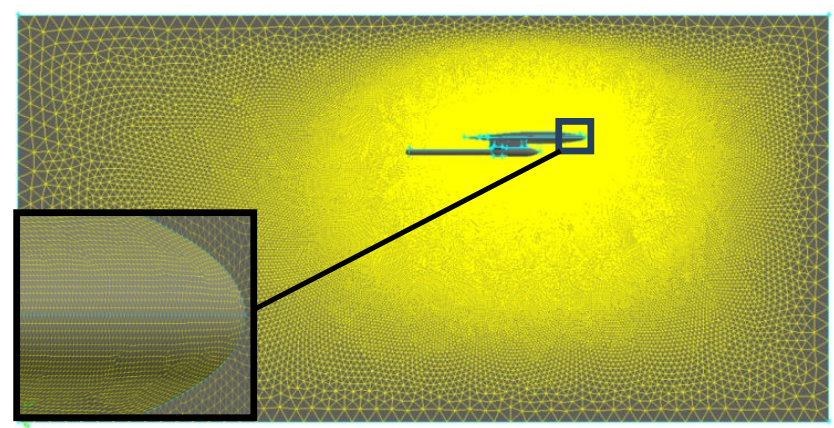

Fig. 4 Wing-store configuration surface meshed with tri elements

The unstructured dynamic mesh method suitable for larger distance moving boundary is the iterative method. Unstructured dynamic mesh is updated by combining spring smoothing and local re-meshing [29].

The object moves as a rigid body, all nodes and boundaries/walls associated with it move as one, the edges between any two mesh nodes are idealized as a network of interconnected springs. A displacement at a given boundary node will generate a force proportional to the displacement along all the springs connected to the node. Using Hook's Law, the force on a mesh node can be written as

$$
\overrightarrow{\mathrm{F}_{1}}=\sum_{\mathrm{j}}^{\mathrm{n}_{\mathrm{i}}} \mathrm{k}_{\mathrm{ij}}\left(\Delta \overrightarrow{\mathrm{x}}_{\mathrm{j}}-\Delta \overrightarrow{\mathrm{x}}_{\mathrm{i}}\right)
$$

where,

$$
k_{i j}=\frac{k_{f a c}}{\sqrt{\left|\vec{x}_{i}-\vec{x}_{j}\right|}}
$$

At equilibrium, the net force on a node due to all the springs connected to the node must be zero. This condition results in an iterative equation

$$
\Delta \vec{x}_{i}^{m+1}=\frac{\sum_{j}^{n_{i}} k_{i j} \Delta \vec{x}_{j}^{m}}{\sum_{j}^{n_{i}} k_{i j}}
$$

In those equations, $x$ is nodal position, $i$ or $j$ is node number, $\mathrm{n}$ is time step and $\mathrm{m}$ is the iteration number.

\section{V.RESULTS AND DiSCUSSIONS}

CFD analyses were performed for three mesh sizes of a wing-pylon-finned store configuration at 0.95 Mach number and $0^{\circ}$ angle of attack during 0.32 seconds using ANSYS FLUENT solver. The predicted store release trajectory and the pressure coefficient distributions on the wing upper and lower surfaces at six chord-wise sections and on the store surface at the angular locations $5^{\circ}, 185^{\circ}, 95^{\circ}$ and $275^{\circ}$ are compared with the experimental data.

The computations are started from $\mathrm{t}=0 \mathrm{~s}$ for obtaining the aerodynamic forces and moments then the solver is coupled with a 6-DOF code for the complete store trajectory prediction using quasi-steady approach. There are both forward and aft ejector forces acting on the store, which will be turned off after the ejector stroke lengths are exceeded. The store inertial characteristics and the constant ejector forces applied on the store are tabulated in Table I.

TABLE I

STORE INERTIAL/MASS AND EJECTOR PARAMETERS

\begin{tabular}{lll}
\hline \hline \multicolumn{1}{c}{ Characteristic Magnitudes } & \multicolumn{1}{c}{ Values } & \multicolumn{1}{c}{ Unit } \\
\hline Mass $(\mathrm{m})$ & 907 & $\mathrm{~kg}$ \\
Center of gravity $\left(\mathrm{x}_{\mathrm{CG}}\right)$ & 1417 (aft of store nose) & $\mathrm{mm}$ \\
Roll Inertia $\left(\mathrm{I}_{\mathrm{XX}}\right)$ & 27 & $\mathrm{~kg} \cdot \mathrm{m}^{2}$ \\
Pitch Inertia $\left(\mathrm{I}_{\mathrm{YY}}\right)$ & 488 & $\mathrm{~kg} \cdot \mathrm{m}^{2}$ \\
Yaw Inertia ( $\left.\mathrm{I}_{\mathrm{ZZ}}\right)$ & 488 & $\mathrm{~kg} \cdot \mathrm{m}^{2}$ \\
Forward Ejector Location $\left(\mathrm{L}_{\mathrm{FE}}\right)$ & 1237.5 (aft of store nose) & $\mathrm{mm}$ \\
Forward Ejector Force $\left(\mathrm{F}_{\mathrm{FE}}\right)$ & 10.7 & $\mathrm{kN}$ \\
Aft Ejector Location $\left(\mathrm{L}_{\mathrm{AE}}\right)$ & 1746.5 (aft of store nose) & $\mathrm{mm}$ \\
Aft Ejector Force $\left(\mathrm{F}_{\mathrm{AE}}\right)$ & 42.7 & $\mathrm{kN}$ \\
Ejector Stroke Length $\left(\mathrm{L}_{\mathrm{ES}}\right)$ & 100 & $\mathrm{~mm}$ \\
\hline \hline
\end{tabular}


The obtained results are presented as follows

\section{A. Surface Pressure Distributions}

\section{Store Surface Pressure}

Fig. 5 shows a comparison between the predicted pressure coefficient distributions and the experimental data along the non-dimensional axis of symmetry length $\mathrm{x} / \mathrm{L}$ at the angular locations of 5, 95, 185, and $275 \mathrm{deg}$ for the fine, medium and coarse grid. There are some discrepancies between the fine grid solutions and the available data due to inviscid nature of the solver. However, for all angular section cuts, the fine and mid grid results are in better agreement with the data than the coarse mesh solutions.
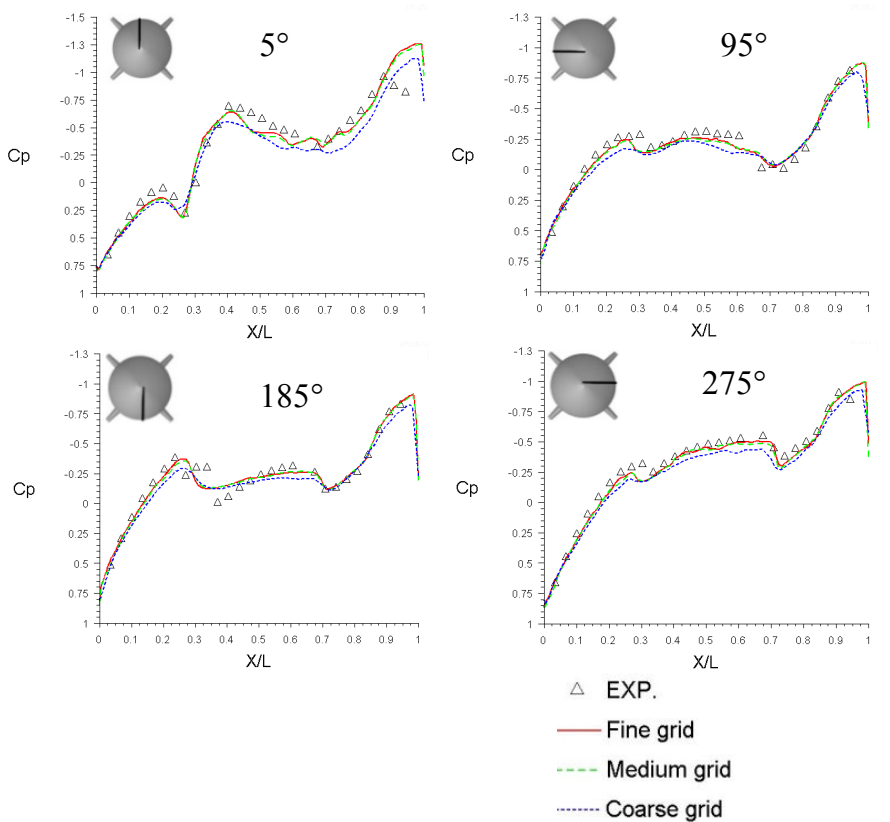

Fig. 5 Store angular pressure coefficient

\section{Wing Surface Pressure}

The pressure data on the wing upper and lower surfaces are extracted at six chord-wise sections, three inboard and three outboard locations as shown in Fig. 6 for comparing the wing pressure distribution before store release and after 0.32 seconds after release.

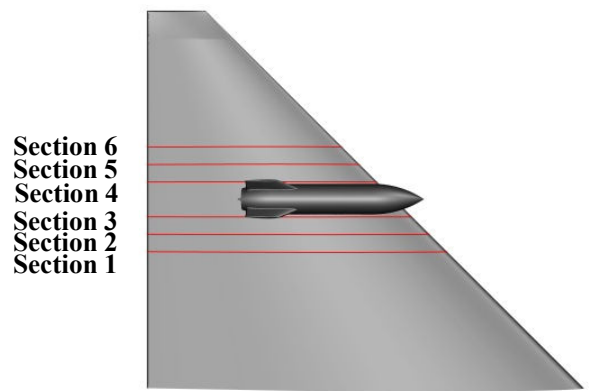

Fig. 6 Pressure measurement sections on the wing

Fig. 7 shows the Cp computational results at six chord-wise sections before store release and after 0.32 seconds after release. Strong variations are noticed in the lower wing surface from $\mathrm{X} / \mathrm{L}=0.2$ to 0.7 where the presence of the store influences the air flow field environment around the wing during separation.
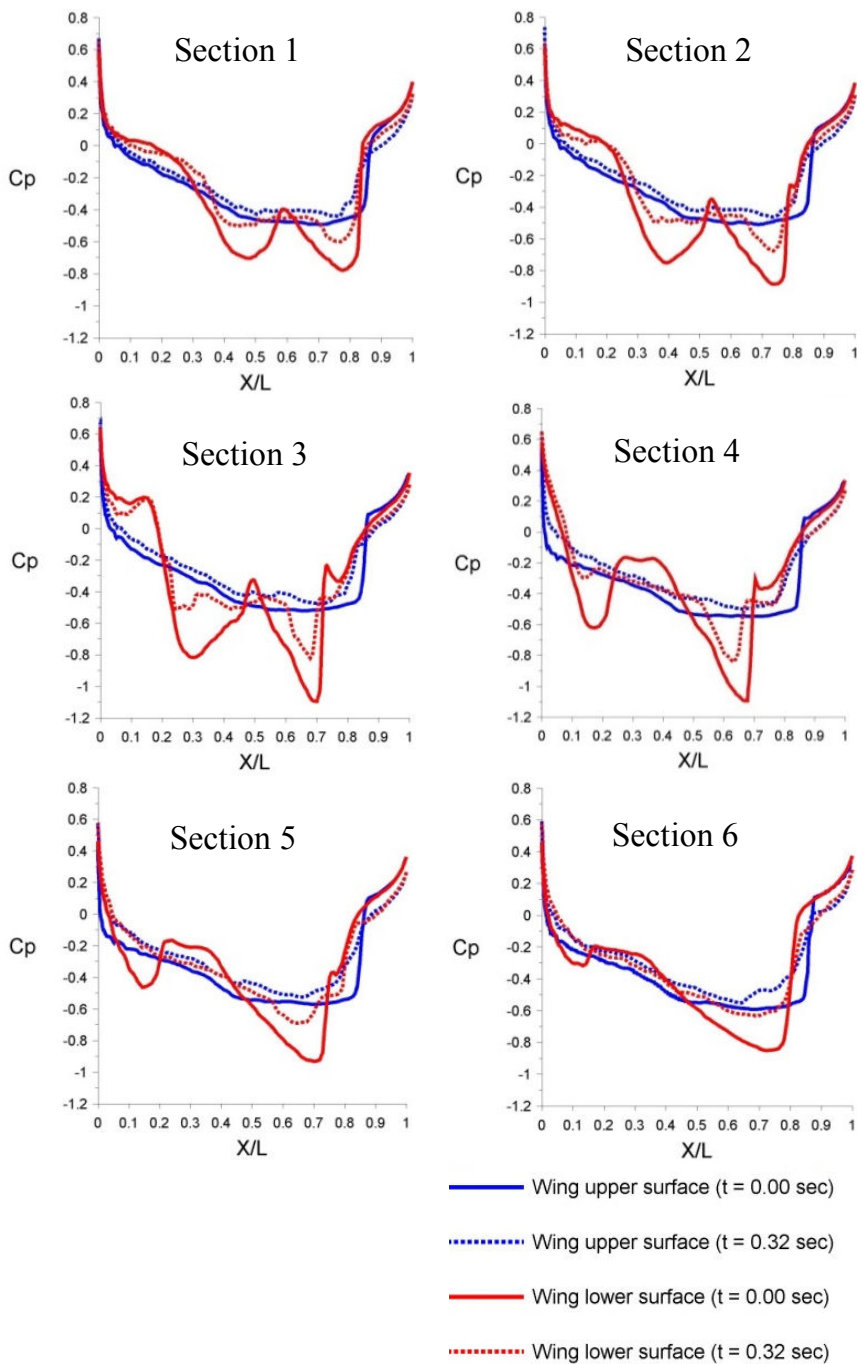

Fig.7 Wing sectional pressure coefficient at time $(\mathrm{t}=0 \& 0.32 \mathrm{sec})$

\section{B. Trajectory Results}

Wing-pylon-finned store test case [1] is solved using the ANSYS FLUENT code with unstructured dynamic grid methodology. The results given in this section represent the linear and angular displacements as well as the velocities, store angle of attack and Sideslip Angle. The trajectory results are presented as follows

\section{Linear and Angular Displacements}

Linear displacements graph are drawn with respect to the store's center of gravity location at the carriage position and given in Fig. 8 store moves backward due to drag force and downward with the effect of gravity and the ejector forces. The store is initially slightly moved towards wing inboard, but soon begins to move slowly outboard with the wing outwash effect. Fine, medium and coarse grids are matches very closely with the experimental data for all displacements in three directions. 
Angular displacements graph are drawn with respect to the coordinate system originated at the store's center of gravity location and given in Fig. 8 total forces acting on the store result in a pitch up, yaw and roll are towards to the outboard of the wing.

The store initially pitches up due to the ejector forces acting on the store for a real time of $\mathrm{t}=0.06$ seconds. After the effect of the ejector forces vanishes, aerodynamic forces acting on the store take the control and result in a pitch down moment on the store. The maximum pitch up angle calculated using fine, medium and coarse grid are (3.9, 3.9 and 3.8 degrees respectively) whereas experimental data shows a maximum of 5.3 degrees. Store rolls towards the outboard side of the wing after its release. The trend of the curve is almost the same with a shift in values from the experimental data. The maximum discrepancy between fine, medium and coarse grid and the test data are $(1.78,1.94$ and 2.69 degrees at time $t=0.24,0.29$ and 0.33 seconds respectively). The store yawing motion is towards to the outboard of the wing. The curve of Fine, medium and coarse grids are matches very closely with the experimental data.

$$
\begin{aligned}
& \triangle \text { CGx EXP } \\
& \text { - CGx Fine grid } \\
& \text {-.. CGx Medium grid } \\
& \text {.... CGx Coarse } \\
& \begin{array}{l}
\text { CGy EXP } \\
\text { CGy Fine grid }
\end{array} \\
& \text { - =- CGy Medium grid } \\
& \text {..... CGy Coarse grid } \\
& \triangle \text { CGz EXP } \\
& - \text { CGz Fine grid } \\
& \text {... CGz Medium grid } \\
& \text {...... CGz Coarse grid } \\
& \triangle \quad \stackrel{\varphi(\text { Roll) EXP }}{\varphi(\text { Roll) Fine grid }} \\
& \varphi \text { (Roll) Fine grid } \\
& \begin{array}{l}
\varphi \text { (Roll) Medium grid } \\
\varphi \text { (Roll) Coarse grid }
\end{array} \\
& \psi \text { (Yaw) EXP } \\
& \psi \text { (Yaw) Fine grid } \\
& \psi \text { (Yaw) Medium gric } \\
& \begin{array}{l}
\Psi \text { (Yaw) Coarse } \\
\theta \text { (Pitch) EXP }
\end{array} \\
& \begin{array}{l}
\theta \text { (Pitch) EXP } \\
\theta \text { (Pitch) Fine grid }
\end{array} \\
& \text {-.- } \theta \text { (Pitch) Medium grid }
\end{aligned}
$$
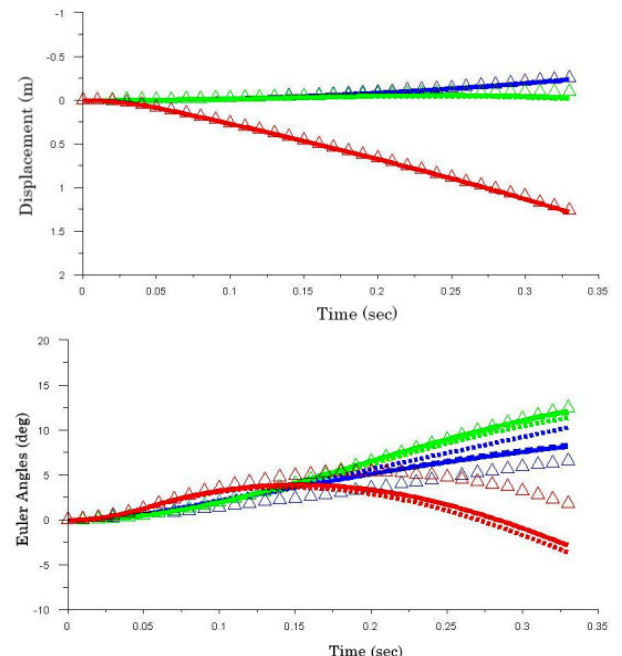

Fig. 8 Comparison of predicted linear and angular displacements with the test data

From the previous results the fine and medium grid gives approximately the same results and in good agreement with the experimental data for yaw and roll angles when compared with the pitch angle. The separation trajectory of the experimental and numerical (fine grid) study is compared and shown in Fig. 9.

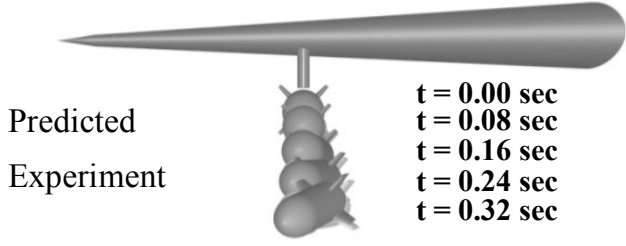

(a) Front view

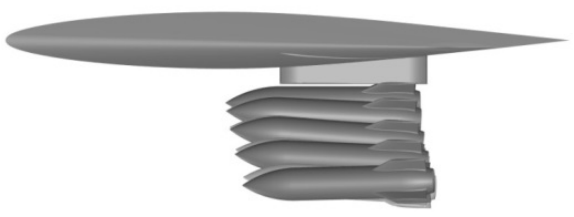

(b) Side view

Fig. 9 Comparison of predicted separation trajectory with the test data

\section{Linear and Angular Velocities of the Store}

Linear and angular velocities of the store along its trajectory are given in Fig. 10. The linear velocity results are in better agreement with the experimental data except for backward velocity there is maximum discrepancy of $0.49,0.5$ and 0.48 $\mathrm{m} / \mathrm{s}$ between the experimental data and the fine, medium and coarse grid respectively, where the velocity is linearly increasing in the negative $\mathrm{x}$ direction, but the trend is almost the same.

A computed angular velocity shows some discrepancy from the experimental data in the angular roll and pitch rate. The angular pitch rate starts to decrease after $\mathrm{t}=0.06 \mathrm{~s}$, corresponding to the end of the stroke. The discrepancies between the angular roll and pitch rate and the available experimental data can be seen after $\mathrm{t}=0.06 \mathrm{~s}$, but the trend of the curve is almost the same with a shift in values from the experimental data.

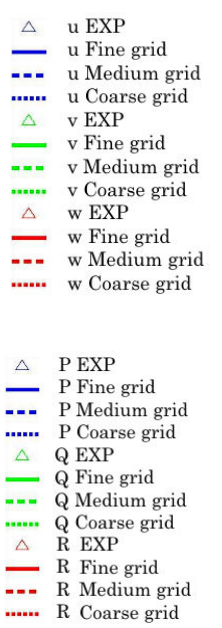

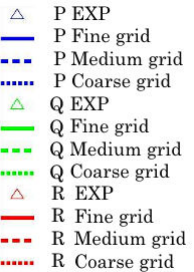

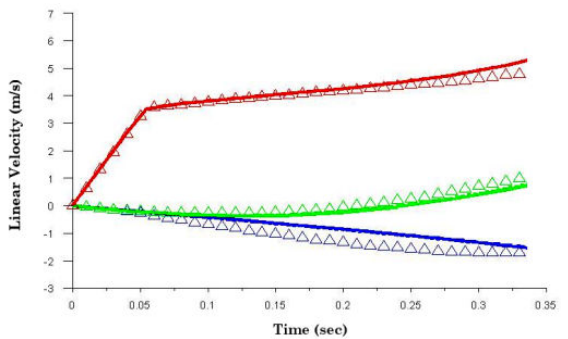

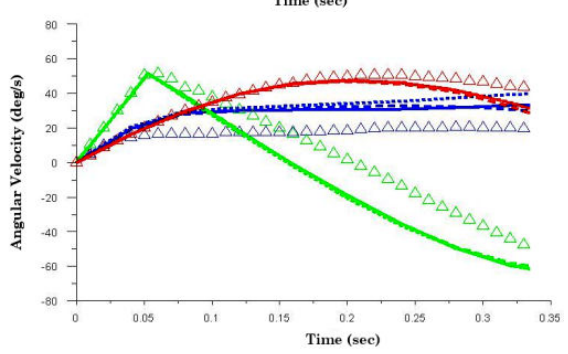

Fig. 10 Comparison of predicted linear and angular displacements with the test data

\section{Linear and Angular Velocities of the Store}

The relative position of the system Oxyz attached to the bomb (the Oxz plane coinciding with the symmetry plane of the wing) and the system Oxayaza attached to the air flow (the Oxa axis is directed along the flight velocity vector VO, and the Oza axis lies in the symmetry plane of the aircraft and is directed downwards) is described by the angle of attack $\alpha$ and sideslip angle $\beta$ as shown in Fig. 11 


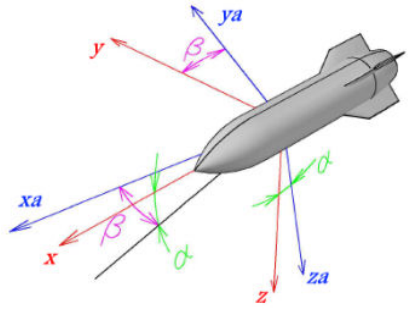

Fig. 11 Coordinate system attached to the store body and airflow

In Fig. 12 the angle of attack increases due to store pitching up motion until $\mathrm{t}=0.22$ seconds, then starts decreasing. The maximum angle of attack calculated is 5 degrees whereas experimental data shows a maximum of 6.5 degrees. The discrepancy from the data starts at time $t=0.13$ seconds. But the trends of the curves are very similar. A sideslip angle shows a very good agreement with the experimental data. In which, store has a negative sideslip angle after its release due to store outboard yawing motion.

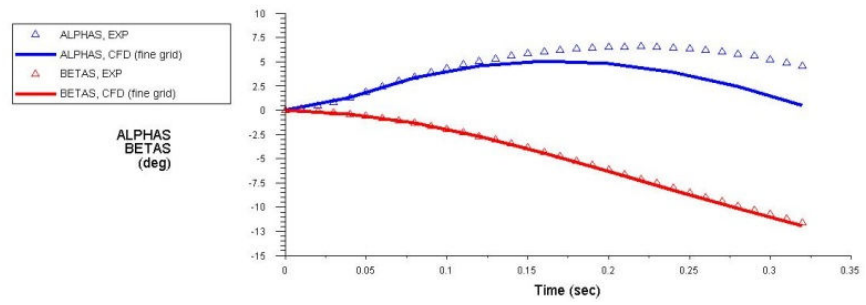

Fig. 12 Comparison of predicted angle of attack $\alpha$ and sideslip angle $\beta$ with the test data

\section{CONCLUSION}

In this paper, finned store separation trajectory from wingpylon-finned store configuration is studied using a dynamic unstructured tetrahedral mesh approach to numerically solving the discretized three dimensional, inviscid and compressible Euler equations. The method used for computations of separation of an external store assuming quasi-steady flow condition. Computations of quasi-steady flow have been directly coupled to a 6DOF rigid-body motion code to generate store trajectories. The computational results are validated against the available experimental data of a generic wing-pylon-store configuration at Mach 0.95.

The pressure coefficient distribution on the store is compared with the available experimental data. Both fine and medium grid show a very good agreement with the experimental data. This shows that, the dynamic unstructured tetrahedral mesh approach works fine with the Euler solver and the pressure distribution on the store is highly affected with this grid node number.

The pressure data on the wing upper and lower surfaces are studied before store release and after 0.32 seconds after release. The results shows strong variations in the lower wing surface from $\mathrm{X} / \mathrm{L}=0.2$ to 0.7 where the presence of the store influences the air flow field environment around the wing during separation.

The trajectory results show that, store moves backward due to drag force and downward with the effect of gravity and the ejector forces. The store is initially slightly moved towards wing inboard, but soon begins to move slowly outboard with the wing outwash effect. The store initially pitches up due to the ejector forces acting on the store after the effect of the ejector forces vanishes, aerodynamic forces acting on the store take the control and result in a pitch down moment on the store, also the store rolls and yaws towards the outboard side of the wing.

Store angle of attack and sideslip angle results show that, the angle of attack increases due to store pitching up motion until $\mathrm{t}=0.22$ seconds, then starts decreasing. Also, store has a negative sideslip angle after its release due to store outboard yawing motion.

No doubt that error is introduced to the results since the solution is obtained without the effects of viscosity. Ejector force approximation could also affect the Euler angles and rates since the correct ejector force modeling used in the experiment is not known exactly. Generally speaking, unstructured dynamic meshing and quasi-steady Euler calculations can be an effective and successful tool for modelling transonic store separation.

\section{REFERENCES}

[1] Rolland H. E., CFD Wing/Pylon/Finned Store Mutual Interference Wind Tunnel Experiment, AEDC-TSR-91-P4, (AD-B152669), USA, 1991.

[2] Carman J. B., Hill D. W. and Christopher J. P., Store Separation Testing Techniques at the Arnold Engineering Development Center, Volume II: Description of Captive Trajectory Store Separation Testing in the Aerodynamic Wind Tunnel (4T), AEDC-TR-79-1, Vol. II (ADA087561), USA, 1980.

[3] Arnold R. J. and Epstein C. S., AGARD Flight Test Techniques Series on Store Separation Flight Testing, AGARDograph No.300, Vol. 5, NATO, 1986.

[4] Mohamed Y., Munikrishna N., Parthiban A. and Balakrishnan N., "Demonstration of Automated CFD Process using Mesh-less Technology", "Proceedings of the 6th European Conference on Computational Fluid Dynamics, ECFD VI", July 20-25, 2014.

[5] Saurabh P. and Bharat R., "Store Separation Simulation using Oct-tree Grid Based Solver", "Symposium on Applied Aerodynamics and Design of Aerospace Vehicles, SAROD 2009”, December 10-12, 2009.

[6] Scott M., Michael J. and Marsha J., "Simulations of 6-DOF Motion with a Cartesian Method", “AIAA Paper 2003-1246", January 6-9, 2003.

[7] Lei T., Jun Y. and Jae L., "Hybrid Cartesian Grid/Gridless Algorithm for Store Separation Prediction", "AIAA Paper 2010-508", 4 - 7 January 2010.

[8] Thorigny P., Bailly D. and Denis P., "Store Separation Trajectory Simulations using "MISSILE" Onera Semi-empirical Aero-prediction Code", "4th European Conference For Aerospace Science, EUCASS", 2011.

[9] Thorsten S., "RANS Simulations with One and Six Degrees of Freedom Rigid Body Motions", "New Research in Numerical and Experimental Fluid Mechanics", Volume 96, pp. 178-185, 2007.

[10] Nathan C. P., Davy M. B., Wei S., "Parallel Computing of Overset Grids for Aerodynamic Problems with Moving Objects", "Progress in Aerospace Sciences", Volume 36, pp. 117-172, 2000.

[11] Carroll F. D., "Development of Chimera Grid Scheme with Applications to Unsteady Problems", "PhD Thesis, Stanford University", USA, 1985.

[12] Demir, $\square$ zgur H., "Computational Fluid Dynamics analysis of Store Separation", "Master Thesis, the Middle East Technical University", 2004.

[13] Mahmood T., Aizud M. N., Zahir S., "Aerodynamic Effects of the Store Release on the Roll Attitude of a Wing Configuration in Transonic Flight", "Proceedings of International Bhurban Conference on Applied Sciences \& Technology”, Islamabad, Pakistan, 10 - 13 January, 2011.

[14] Young M. J., Young S. K., Jae W. L., Young H. B., and Jun S. P., "Aerodynamic Analysis on the Supersonic Separation of Air-Launching 
Rocker from the Mother Plane", "International Conference on Computational Science and Its Applications", LNCS 3984, pp. 457 466, 2006.

[15] George Z., Michael T. C., Daniel J. B., Eric S., and Mark D. B., “A Toolkit for Parallel Overset Grid Assembly Targeting Large-Scale Moving Body Aerodynamic Simulations", "Proceedings of the 19th International Meshing Roundtable", pp 385-401, 2010.

[16] Mun S. J. and Oh J. K., "A Parallel Unstructured Overset Mesh Technique for Unsteady Flow Simulations", "Proceedings of the Fourth International Conference of Computational Fluid Dynamics", 2006.

[17] Junfeng G., Zhou Z., Bin L., "Using the Unstructured Dynamic Mesh to Simulate Multi-Store Separating from Aircraft", "Journal of Procedia Engineering", Volume 16, pp. 572 - 580, 2011.

[18] Ingemar P., Anders L., "Transonic Store Separation Studies on the SAAB GRIPEN Aircraft Using Computational Aerodynamics", "26th International Congress of the Aeronautical Sciences", Alaska, USA, 14 19 September, 2008

[19] Radha K. P., Mahesh M. S., "Store Separation Analysis Using Computational Fluid Dynamics", "Proceedings of IRF International Conference", Pune, India, 30th March, 2014.

[20] Jafari M., Toloei A., Ghasemlu S., Parhizkar H., "Simulation of Store Separation using Low-cost CFD with Dynamic Meshing", "International Journal of Engineering", Volume 27, No. 5, pp. 775-784, 2014.

[21] Rakesh K., "parametric study on Trajectory of Released Store", "16th Annual CFD Symposium”, Bangalore, 11-12 August, 2014.

[22] Yunus E. S., Emrah G., Ali A., "Numerical Simulations of Store Separation Trajectories Using the EGLIN Test", "Scientific Technical Review", Volume 63, No.1, pp. 10-16, 2013.

[23] Torsten B., Lars T., "Numerical Investigation of the Impact of Maneuver on Store Separation Trajectories", “AIAA Paper 2010-4241", 28 June 1 July, 2010.

[24] Torsten B., "Numerical Simulation of Store Separation for Quasi-Steady Flow", "FOI Technical Paper No.FOI-R-2761-SE”, Stockholm, 2009.

[25] Raghavender V., Kannan R., "Computational Aerodynamic Analysis on Store Separation from Aircraft using Pylon", "International Journal of Engineering Science Invention", pp. 27-31, 2014.

[26] Elias E. P., Spyridon D. K., "CFD Transonic Store Separation Trajectory Predictions with Comparison to Wind Tunnel Investigations", "International Journal of Engineering", Volume 3, No.6, pp. 538-553, 2010.

[27] Tomaro R. F., Witzeman F. C. and Strang W. Z., "A Solution on the F$18 \mathrm{C}$ for Store Separation Simulation Using Cobalt60", "AIAA Paper 99$0122 ", 1999$.

[28] Mark W. J., “A novel Cartesian CFD cut cell approach”, “Journal of Computers \& Fluids", Volume 79, pp. 105-119, 2013

[29] Fluent User's Guide, Version 15, ANSYS, Inc., April 2014. 